$\mathrm{E}$

EVALUAR
2018, Vol. 18, No 2.

ISSN 1667-4545

Recuperado de https://revistas.unc.edu.ar/index.php/revaluar

Laboratorio de Evaluación Psicológica y Educativa

Facultad de Psicología - Universidad Nacional de Córdoba

\title{
Adaptación y validación de un instrumento para evaluar el constructo compasión
}

\section{Adaptation and validation of an instrument to evaluate the compassion construct}

\author{
María Carolina Klos * 1,2, Viviana Noemí Lemos ${ }^{1,3}$ \\ 1 - Centro Interdisciplinario de Investigación en Psicología Matemática y Experimental Dr. Horacio J. A. Rimoldi (CIIPME)- \\ Consejo Nacional de Investigaciones Cientificas y Técnicas (CONICET), Buenos Aires, Argentina. \\ 2 - Universidad Adventista del Plata, Centro de Investigaciones en Psicología y Ciencias Afines, \\ Libertador San Martín, Entre Ríos, Argentina. \\ 3 - Pontificia Universidad Católica Argentina, Facultad Teresa de Ávila, Paraná, Entre Ríos, Argentina. \\ Introducción \\ Método \\ Resultados \\ Discusión \\ Referencias
}

Recibido: 04/01/2018 Revisado: 30/01/2018 Aceptado: 26/02/2018

\section{Resumen}

El presente estudio tuvo por objetivo la traducción y validación de The Compassion Of Others' Lives scale (COOL; Escala de Compasión hacia Otros; Chang, Fresco, \& Green, 2014), evaluando su funcionamiento en una población de adolescentes y adultos jóvenes argentinos. El estudio empírico instrumental se realizó a partir de un muestreo no probabilístico, con la participación de 385 adolescentes y adultos jóvenes, 210 mujeres y 175 varones de 13 a 23 años $(\mathrm{M}=17.32 ; \mathrm{DE}=2.51)$. Para estudiar las propiedades psicométricas de la versión propuesta, se analizó el poder discriminativo de los ítems, la consistencia interna del instrumento, sumando diferentes evidencias de validez de constructo, convergente y de criterio. La versión traducida y adaptada de la COOL presentó propiedades psicométricas satisfactorias, por lo cual resulta factible de ser utilizada para evaluar el constructo compasión en adolescentes $\mathrm{y}$ adultos jóvenes, con un adecuado grado de fiabilidad y validez en el contexto en que fue estudiada.

Palabras Clave: Compasión, evaluación psicológica, adolescencia, psicología positiva

\begin{abstract}
The present study aimed to translate and validate "The Compassion of Others' Lives Scale (COOL; Chang, Fresco, \& Green, 2014)", evaluating its functioning in a population of Argentine adolescents and young adults. The empirical instrumental study was carried out in a non-probabilistic sample, consisting of 385 adolescents and young adults, 210 women and 175 men from 13 to 23 years of age $(\mathrm{M}=17.32, \mathrm{SD}=2.51)$. To study the psychometric properties of the proposed version, we analyzed the following: the discriminative power of the items, the internal consistency of the instrument, adding different evidences of construct, convergent, and criterion validity. The translated and adapted version of COOL showed satisfactory psychometric properties, so it is feasible to be used to evaluate the compassion construct in adolescents and young adults, with an adequate degree of reliability and validity in the context in which it was studied.
\end{abstract}

Keywords: Compassion, psychological evaluation, adolescence, positive psychology

*Correspondencia a: M. C. Klos, Dirección Postal: Ramos Mejía 284, Libertador San Martín (3103), Entre Ríos, Argentina. Celular: 0343-155064263.

e-mail: mcarolinaklos@gmail.com

Cómo citar este artículo: Klos, M. C., \& Lemos, V. N. (2018). Adaptación y validación de un instrumento para evaluar el constructo compasión. Revista

Evaluar, 18(2), 31-44. Recuperado de https://revistas.unc.edu.ar/index.php/revaluar 


\section{Introducción}

A lo largo de los años la compasión ha sido una temática estudiada ampliamente tanto en Oriente como en Occidente (Poch \& Vicente, 2010). Asimismo, dicha temática ha sido abordada desde distintas áreas como la filosofía, psicología positiva y social, neuropsicología, pedagogía y espiritualidad. Los estudios en estas líneas de trabajo resultan en aportes complementarios para entender la compasión.

Dado que la compasión ha sido considerada una experiencia afectiva, distintos teóricos incluyen su estudio en el contexto de las emociones morales (Díaz-Alzate, Arbeláez-Gómez, \& David-Manco, 2015; Mercadillo \& Díaz, 2013; Mercadillo, Díaz, \& Barrios, 2007; OstroskySolís \& Vélez-García, 2008; Saavedra, Mola, Gancedo, Belaus, \& Reyna, 2013). Las emociones morales están conformadas por relaciones y juicios entre uno mismo y los demás. Además, se las asocia a intereses de bienestar social y personal, es decir, dependen en mayor medida de la cultura, contexto social y de una evaluación previa a su manifestación, lo cual las diferencia de las emociones básicas al extenderse más allá de la esfera inmediata de uno mismo (Kroll \& Egan, 2004; Mercadillo et al., 2007; Ostrosky-Solís \& Vélez-García, 2008).

En el estudio de las emociones morales fueron propuestas cuatro subdivisiones: emociones de condena, emociones de autoconciencia, emociones relativas al sufrimiento ajeno y emociones de admiración. Dentro de la familia de emociones morales desencadenadas por la percepción del sufrimiento ajeno, se encuentra la compasión, definida como una respuesta emotiva a un juicio valorativo acerca del padecimiento de una persona (Haidt, 2003; Mercadillo et al., 2007).

Por otro lado, Lazarus (1991) propone que las emociones se dividen en seis categorías: emo- ciones desagradables, emociones existenciales, emociones provocadas por condiciones de vida desfavorables y favorables y emociones empáticas. Dentro de esta última categoría se encuentran la gratitud y la compasión. Lazarus y Lazarus (1996) plantean que la compasión involucra la comprensión del estado emocional ajeno, asociada a un deseo de aliviar o reducir el sufrimiento del otro (Regner, 2009).

Los subprocesos dentro de la compasión son notar, sentir y aliviar. Estos, componen un proceso multidimensional tripartito integrado por: el notar el sufrimiento de otra persona, la empatía como conexión cognitiva y afectiva frente al sentimiento de dolor de esa persona y el actuar de una manera destinada a aliviar el sufrimiento, independientemente de que se logre o no (Lilius, Worline, Dutton, Kanov, \& Maitlis, 2011; Miller, 2007). Más recientemente, los autores Jazaieri et al. (2012) incorporaron una nueva dimensión, definiendo la compasión como un proceso multidimensional formado por cuatro componentes principales: una conciencia de sufrimiento (cognición empática), la preocupación simpática relacionada con el ser movido emocionalmente por el sufrimiento del otro (componente afectivo), el deseo de aliviar ese sufrimiento (intención) y una respuesta o disposición a ayudar en el alivio de ese sufrimiento (motivación-acción).

Batson y Moran (1999, en Saavedra et al., 2013), para explicar la compasión, proponen el modelo empatía-altruismo, de acuerdo con el cual la percepción de las necesidades de otra persona puede generar una reacción emocional de empatía, la cual produce un deseo altruista de reducir el malestar. Goetz, Keltner y Simon-Thomas (2010) coinciden en que el altruismo (motivación o acción de ayudar a quien sufre) es otro componente de la compasión, resaltando que no todo acto altruista es realizado luego de experimentar compasión, ya que pueden existir otras motivaciones. 
En lo que respecta al estudio de la ayuda social, cabe destacar la existencia de conceptos estrechamente vinculados pero diferenciables. La conducta prosocial es el concepto más general ya que se refiere a aquellas acciones que benefician a otras personas y engloba, a su vez, múltiples conductas (Caprara, Steca, Zelli, \& Capanna, 2005; Yubero \& Larrañaga-Rubio, 2002). Las conductas de ayuda, tienen que ver con las acciones que buscan responder a una necesidad ajena proporcionando algún beneficio o incrementando el bienestar de otra persona. Por otro lado, el altruismo o conducta altruista incluye acciones que benefician a los demás y que a la vez, suponen una motivación desinteresada por parte de quien realiza la acción (Yubero \& Larrañaga-Rubio, 2002). La compasión, se da en un contexto particular al notar el dolor o malestar de otra persona y brindar una respuesta de alivio motivada por la empatía.

En cuanto a la medición de la compasión, se han desarrollado diversas escalas tales como:

- Compassionate Love Scale (CLS; Escala de Amor Compasivo; Sprecher \& Fehr, 2005), la cual cuenta con 21 ítems autoadministrables y posee dos formas: una relacionada con lo que se experimenta frente a cercanos y otra frente a extraños o la humanidad en general.

- The Compassion Scale (CS-M; Escala de Compasión; Martins, Nicholas, Shaheen, Jones, \& Norris, 2013), evalúa cinco dominios de la compasión: generosidad, hospitalidad, objetividad, sensibilidad y tolerancia en las redes sociales, en tres contextos relacionales: con extraños, familiares y amigos. Esta escala, está conformada por 10 ítems autoadministrables.

- The Compassion Scale (CS-P; Escala de Compasión; Pommier \& Neff, 2010), posee 24 ítems autoadministrables y contempla seis elementos opuestos (amabilidad-indiferencia, atención-despreocupación y contacto-separación).
- Compassionate Care Assessment Tool (CCAT; Herramienta de Evaluación de Cuidados Compasivos; Burnell \& Agan, 2013), la cual evalúa mediante 28 ítems los niveles de compasión del personal de enfermería en hospitales; , a diferencia de las otras escalas mencionadas, debe ser contestada por los pacientes respondiendo desde la percepción que ellos tienen de sus cuidadores.

Otro de los instrumentos utilizados para la evaluación de la compasión es

- The Compassion of Others 'Lives Scale (COOL; Escala de Compasión hacia Otros; Chang, Fresco, \& Green, 2014), compuesta por 26 ítems autoadministrables. Esta escala se desprende del modelo teórico empatía-altruismo para explicar la compasión (Chang et al., 2014), que contempla el componente cognitivo-afectivo (empatía) y el acto altruista en dirección a aliviar o aminorar el malestar del otro (altruismo). Sin embargo, el instrumento operacionaliza las dimensiones de empatía y conducta de alivio, quedando excluida la evaluación de las motivaciones altruistas.

Se considera que The Compassion of Others' Lives Scale (la Escala de Compasión hacia Otros), a diferencia de las otras herramientas de evaluación mencionadas con anterioridad, es la más pertinente y consistente en relación con a la línea teórica abordada en el estudio de la compasión. Chang et al. (2014), han reportado evidencias de la validez y confiabilidad de la escala en una población norteamericana de entre 18 y 43 años. A su vez, los aportes de Coskun, Kavakli, Babayigit y Chang (2017) han permitido conocer el funcionamiento del instrumento en una cultura oriental, al evaluar las propiedades psicométricas del mismo en una muestra de adultos de Turquía. Los resultados arrojados también evidenciaron un funcionamiento adecuado en dicha población.

En este marco, y considerando la importancia de utilizar instrumentos adecuadamente validados al contexto en que serán utilizados cuando 
los mismos han sido diseñados en otro idioma y bajo otras condiciones culturales, (Fernández, Pérez, Alderete, Richaud, \& Fernández-Liporace, 2011; Muñiz, Elosua, \& Hambleton, 2013; Richaud de Minzi, Lemos, \& Oros, 2003), el presente estudio tuvo como objetivo principal la traducción y validación del instrumento The Compassion of Others' Lives Scale (Escala de Compasión hacia Otros), evaluando su funcionamiento en una población de adolescentes y adultos jóvenes argentinos.

\section{Método}

Dados los objetivos del estudio y los análisis estadísticos realizados para su consecución, se desarrolló un estudio de tipo Instrumental (Gibbons, Flores de Arévalo, \& Mónico, 2004; Montero \& León, 2007; Servera \& Cardo, 2006).

\section{Participantes}

Se trabajó con una muestra no probabilística intencional de 385 adolescentes y adultos jóvenes, de los cuales 210 fueron mujeres $(54.5 \%) \mathrm{y}$ 175 fueron varones (45.5\%) de 13 a 23 años de edad $(\mathrm{M}=17.32$; $\mathrm{DE}=2.51)$. De los participantes, 158 fueron encuestados en una universidad privada de la provincia de Entre Ríos, 129 eran alumnos de un colegio secundario de la misma provincia y 94 de un colegio secundario de la provincia de Santa Fe. Por lo tanto, la muestra quedó conformada por 223 estudiantes de secundaria y 158 estudiantes universitarios.

\section{Instrumentos}

Cuestionario de datos sociodemográficos. Se administró una encuesta ad hoc que solicitaba a los participantes consignar la edad, el género, el nivel de estudios, el lugar de residencia y la religión.

Versión traducida y adaptada de The Compassion of Others' Lives scale (COOL; Escala de Compasión hacia Otros). La COOL (Chang et al., 2014) está conformada por 26 ítems, de los cuales 13 corresponden a la dimensión de empatía (p. ej., Entiendo los sentimientos de los demás) y los 13 restantes a la dimensión de alivio de sufrimiento (p. ej., Demuestro cariño a personas que están pasando por dolor emocional) presentados en un formato de escala Likert de 7 puntos graduada según el grado de acuerdo con la afirmación (desde 1 = fuertemente en desacuerdo, hasta 7 = fuertemente de acuerdo). La escala permite obtener un puntaje global del grado de compasión hacia otros, como así también un puntaje para cada subescala (empatía y alivio del sufrimiento). Chang et al. (2014) estudiaron las propiedades psicométricas de la COOL en una muestra de 355 sujetos, obteniendo una consistencia interna de $\alpha$ $=.98$ para la subescala empatía y de $\alpha=.90$ para alivio del sufrimiento. La estabilidad de la prueba fue evaluada a través de un test-retest, observándose una $r=.87$ para la sub-escala empatía y de $r=.88$ para alivio del sufrimiento. También fueron evaluadas la validez convergente y la discriminante, encontrando resultados satisfactorios congruentes con la teoría (Chang et al., 2014).

La traducción y adaptación al español de Argentina se hizo respetando la representación del constructo en nuestro contexto, es decir, no se priorizó la traducción literal de los ítems, sino su sentido psicológico, tal como es recomendado en la adaptación de un instrumento de un contexto idiomático y cultural a otro diferente (Muñiz et al., 2013; Muñiz -Fernández, 2000). 
Interpersonal Reactivity Index (IRI; Inventario de reactividad interpersonal; Davis, 1983). El La IRI operacionaliza un constructo multidimensional de empatía (aspectos cognitivos y afectivos) y fue estudiado en población argentina por Richaud de Minzi (2008), encontrando propiedades psicométricas adecuadas. En este trabajo se consideraron las dimensiones de: preocupación empática (emocional) y toma de perspectiva (cognitiva), no obstante, la escala completa incluye además las subescalas de fantasía y malestar personal. Las opciones de respuesta van del 1 al 5 (no me describe bien, me describe un poco, bastante bien, bien y me describe muy bien), siendo el criterio de elección de acuerdo a cuán acertada considera la persona que es la descripción (p. ej., Me preocupa y conmueve la gente con menos suerte que yo).

\section{Procedimientos seguidos para la traducción del instrumento}

Por un lado, se contactó a los autores de la versión original del instrumento solicitando consentimiento para su traducción al español y adaptación en Argentina. Seguidamente, se procedió a realizar la traducción con la colaboración, de forma paralela, de una traductora de inglés y un adulto joven bilingüe. A continuación, la versión preliminar traducida al español fue nuevamente traducida al inglés a modo de asegurar la conservación del sentido de los ítems de la escala original.

Por otro lado, se contactó vía mail a siete jueces expertos en el área de psicología positiva $\mathrm{y}$ mediante un formulario brindaron su valoración respecto a la pertinencia de lo que pretende evaluar cada reactivo del instrumento y también se les solicitó que evalúen la traducción de cada ítem, dando lugar a observaciones o traducciones alternativas.
Procedimientos seguidos

para la recolección de datos

Para el estudio del funcionamiento de la versión traducida del instrumento en adolescentes y adultos jóvenes argentinos, los participantes fueron seleccionados a través de un muestreo no probabilístico intencional. Se contó con el consentimiento informado de todos los sujetos participantes. Los menores, además, contaron con la autorización de sus padres o tutores legales. La participación fue voluntaria garantizando la confidencialidad de la información obtenida.

Los instrumentos fueron administrados de manera colectiva. En el caso de los adolescentes, dichos instrumentos fueron administrados en el contexto escolar, en horas acordadas durante la jornada académica, mientras que en el caso de los adultos jóvenes, fueron administrados en el contexto de una jornada de capacitación organizada por la Universidad Adventista del Plata. Se contó, además, con la previa autorización de los directivos y docentes de las instituciones educativas que participaron.

\section{Procedimientos para el análisis de datos}

Para estudiar las propiedades psicométricas de la versión traducida y adaptada de la COOL, se utilizaron los programas estadísticos AMOS 17 (Arbuckle, 2008) y SPSS 22 (IBM Corp., 2013).

Para evaluar el poder discriminativo de los ítems se utilizó el criterio de grupos contrastantes utilizando la prueba $t$ para muestras independientes. Con el fin de evaluar la validez de estructura interna del instrumento, se realizó un análisis factorial confirmatorio (AFC), mediante el método de estimación de parámetros por máxima verosimilitud. La consistencia interna de la escala fue evaluada mediante el coeficiente alfa de 
Cronbach. También se recogieron evidencias de validez convergente, correlacionando las dimensiones del IRI y la COOL, esperando una correlación positiva y significativa entre ambas variables (Hwang, Plante, \& Lackey, 2008; Pommier \& Neff, 2010). Asimismo, se recogieron datos de validez de criterio y discriminante, correlacionando la COOL con la escala de CP y con la de AFV esperando una correlación positiva y negativa respectivamente, tal como se esperaría teóricamente (Cuello \& Oros, 2014; Regner, 2009).

\section{Resultados}

La versión traducida fue sometida a la valoración de siete jueces argentinos expertos en el tema, quienes partiendo de la definición teórica del constructo compasión y sus dimensiones, evaluaron la adecuación de la traducción. Tuvieron en consideración si los ítems reflejaban de manera clara el constructo a operacionalizar, recogiendo a su vez posibles alternativas de redacción.

A partir de las sugerencias de los jueces, 15 ítems fueron modificados (la mayoría con cambios menores de expresión). Por ejemplo, la alternativa de traducción Sin importar si existe un beneficio o no, estoy dispuesto a ayudar a quien lo necesita fue reemplazada por Estoy dispuesto a ayudar a quien lo necesita sin importar si esto me beneficia. La alternativa Soy bondadoso con los demás, fue reemplazada por Estoy atento a las necesidades de otros, dado que los jueces acordaron que representaba mejor la dimensión empatía; el ítem Ante los sentimientos de otra persona puedo proyectarme y sentir lo mismo, fue modificado por Puedo proyectarme y sentir lo mismo que otras personas.

En cuanto al funcionamiento de los ítems, todos los ítems resultaron discriminativos, como se muestra en la Tabla 1.

Tabla 1

Poder discriminativo de los ítems de la versión final de la escala.

\begin{tabular}{|c|c|c|c|c|c|c|c|}
\hline & \multirow{2}{*}{ Ítems de la escala } & \multicolumn{2}{|c|}{ Grupo bajo } & \multicolumn{2}{|c|}{ Grupo alto } & \multirow{2}{*}{$t$} & \multirow{2}{*}{$p$} \\
\hline & & $M$ & $D E$ & $M$ & $D E$ & & \\
\hline 1. & Siento las emociones de los demás & 4.24 & 1.20 & 6.15 & 1.04 & -11.81 & $<.001$ \\
\hline 2. & Entiendo los sentimientos de las personas & 4.36 & 1.27 & 6.29 & .87 & -12.36 & $<.001$ \\
\hline 3. & $\begin{array}{l}\text { Cuando sé cómo se siente alguien, estoy más dis- } \\
\text { puesto a ayudarlo }\end{array}$ & 5.47 & 1.24 & 6.74 & .55 & -9.33 & $<.001$ \\
\hline 4. & $\begin{array}{l}\text { Siento la obligación de ayudar a quien está pasando } \\
\text { por mucho dolor }\end{array}$ & 4.96 & 1.43 & 6.72 & .58 & -11.39 & $<.001$ \\
\hline 5. & $\begin{array}{l}\text { Si se trata de un amigo/a, siempre estoy disponible } \\
\text { para brindarle mi ayuda }\end{array}$ & 6.25 & .90 & 6.83 & .50 & -5.56 & $<.001$ \\
\hline 6. & Estoy atento a las necesidades de otros & 4.19 & 1.23 & 6.27 & .73 & -14.39 & $<.001$ \\
\hline 7. & $\begin{array}{l}\text { Estoy dispuesto a ayudar a los demás porque eso me } \\
\text { hace sentir bien conmigo mismo }\end{array}$ & 5.00 & 1.49 & 6.69 & .61 & -10.46 & $<.001$ \\
\hline 8. & $\begin{array}{l}\text { Puedo proyectarme y sentir lo mismo que otras } \\
\text { personas }\end{array}$ & 3.95 & 1.33 & 6.17 & .80 & -14.15 & $<.001$ \\
\hline 9. & $\begin{array}{l}\text { Sin importar cuál es la circunstancia, siento la obli- } \\
\text { gación de ayudar a quien parece estar en problemas }\end{array}$ & 4.16 & 1.22 & 6.52 & .68 & -16.75 & $<.001$ \\
\hline
\end{tabular}


10. No puedo evitar sentirme muy mal por una persona que padece hambre

11. Me preocupo cuando veo a personas pasando por dolor o sufrimiento

12. Me genera tristeza ver a alguien que está solo por más que no lo conozca

13. Me gusta ayudar a otros, cuando veo que de esa manera alivio su malestar

14. Considero que soy sensible con lo que le pasa a los demás

15. Me afectan las emociones de otras personas

16. Si me doy cuenta que alguien cercano está pasando por un mal momento emocional, siento la obligación de hablar con él/ella sobre el tema

17. Estoy dispuesto a ayudar a quien lo necesita sin importar si esto me beneficia

18. Me preocupan las personas que se encuentran en una situación peor que la mía

19. Tengo la habilidad de ponerme en el lugar de otros

20. No necesito recompensas por ayudar a otros

21. Naturalmente soy consciente de los sentimientos y emociones de otros

22. Cuando alguien está en riesgo, tiendo a ser el primero en intervenir y ver en qué puedo ayudar

23. Cuando me relaciono con otra persona, me imagino estando en una situación similar

24. Si un extraño tropieza y se cae, ayudaría a levantarlo

25. Le sostendría la puerta a una persona con discapacidad

26. Demuestro cariño hacia personas que están pasando por dolor emocional

\begin{tabular}{|c|c|c|c|c|c|}
\hline 4.34 & 1.57 & 6.35 & 1.00 & -10.65 & $<.001$ \\
\hline 4.77 & 1.30 & 6.71 & .57 & -13.57 & $<.001$ \\
\hline 4.35 & 1.64 & 6.61 & .83 & -12.16 & $<.001$ \\
\hline 4.90 & 1.25 & 6.73 & .49 & -13.56 & $<.001$ \\
\hline 3.86 & 1.29 & 6.27 & .85 & -15.40 & $<.001$ \\
\hline 3.96 & 1.40 & 6.16 & .96 & -12.76 & $<.001$ \\
\hline 4.92 & 1.43 & 6.65 & .64 & -11.04 & $<.001$ \\
\hline 4.91 & 1.39 & 6.70 & .57 & -11.84 & $<.001$ \\
\hline 4.66 & 1.37 & 6.73 & .60 & -13.86 & $<.001$ \\
\hline 4.08 & 1.55 & 6.46 & .67 & -14.10 & $<.001$ \\
\hline 5.79 & 1.40 & 6.70 & .90 & -5.36 & $<.001$ \\
\hline 4.33 & 1.22 & 6.30 & .71 & -13.81 & $<.001$ \\
\hline 3.78 & 1.34 & 6.10 & .81 & -14.65 & $<.001$ \\
\hline 4.03 & 1.49 & 6.46 & .80 & -14.33 & $<.001$ \\
\hline 5.42 & 1.50 & 6.72 & .56 & -8.11 & $<.001$ \\
\hline 6.55 & .85 & 6.94 & .28 & -4.42 & $<.001$ \\
\hline 5.19 & 1.31 & 6.74 & .61 & -10.72 & $<.001$ \\
\hline
\end{tabular}

que en un trabajo preliminar reciente observamos con otra muestra más reducida una estructura unidimensional obtenida a través de un análisis factorial exploratorio (Klos, Lemos, \& Chang, 2017), se corrió un segundo Modelo, evaluando cuál de los dos modelos obtenía un mejor ajuste (ver Figura 1 y Figura 2). mensiones, tal como proponen los autores de la COOL (Chang et al., 2014). Seguidamente, dado 


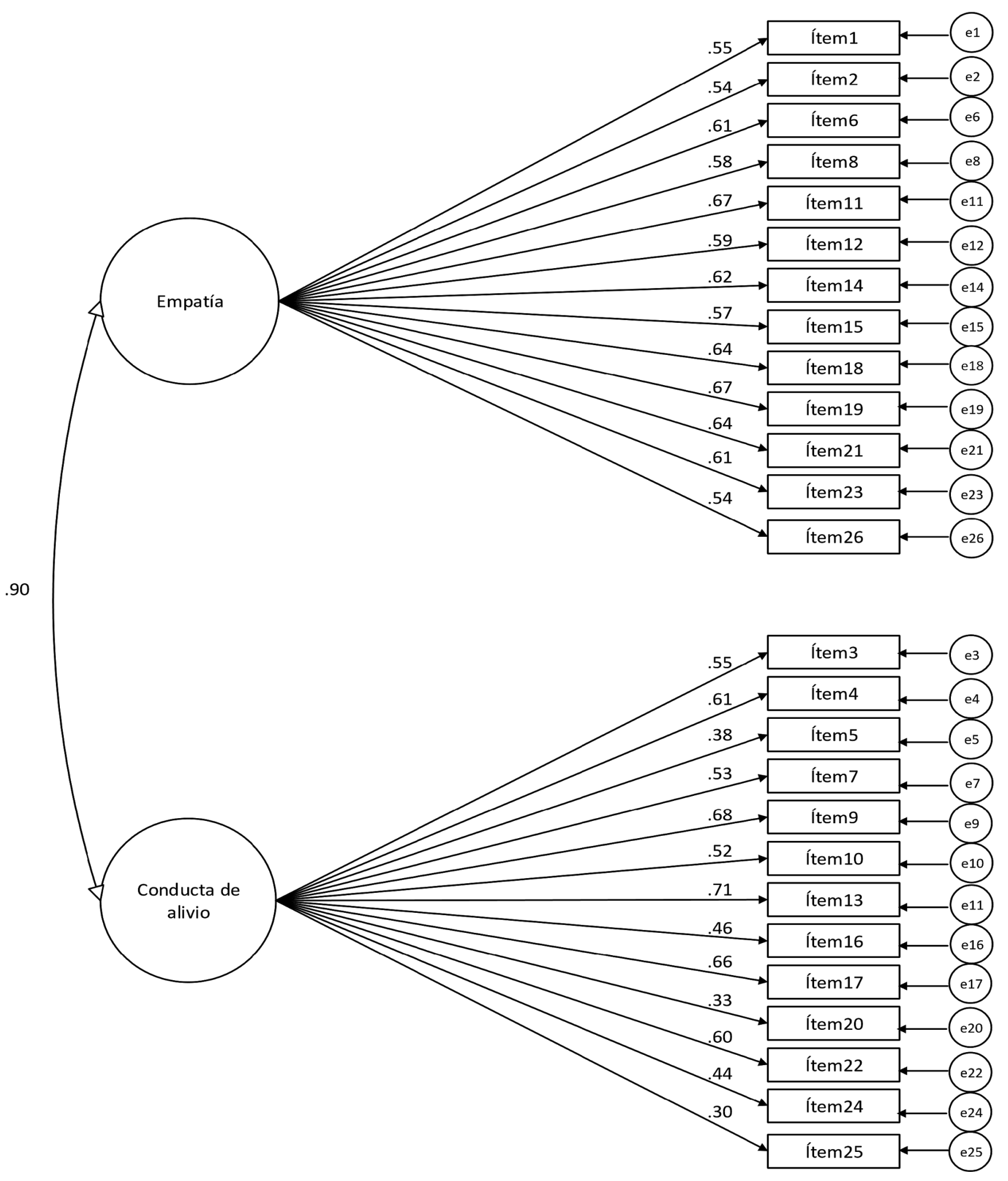

Figura 1

Modelo 1, versión bifactorial (empatía y conducta de alivio) de la COOL. 


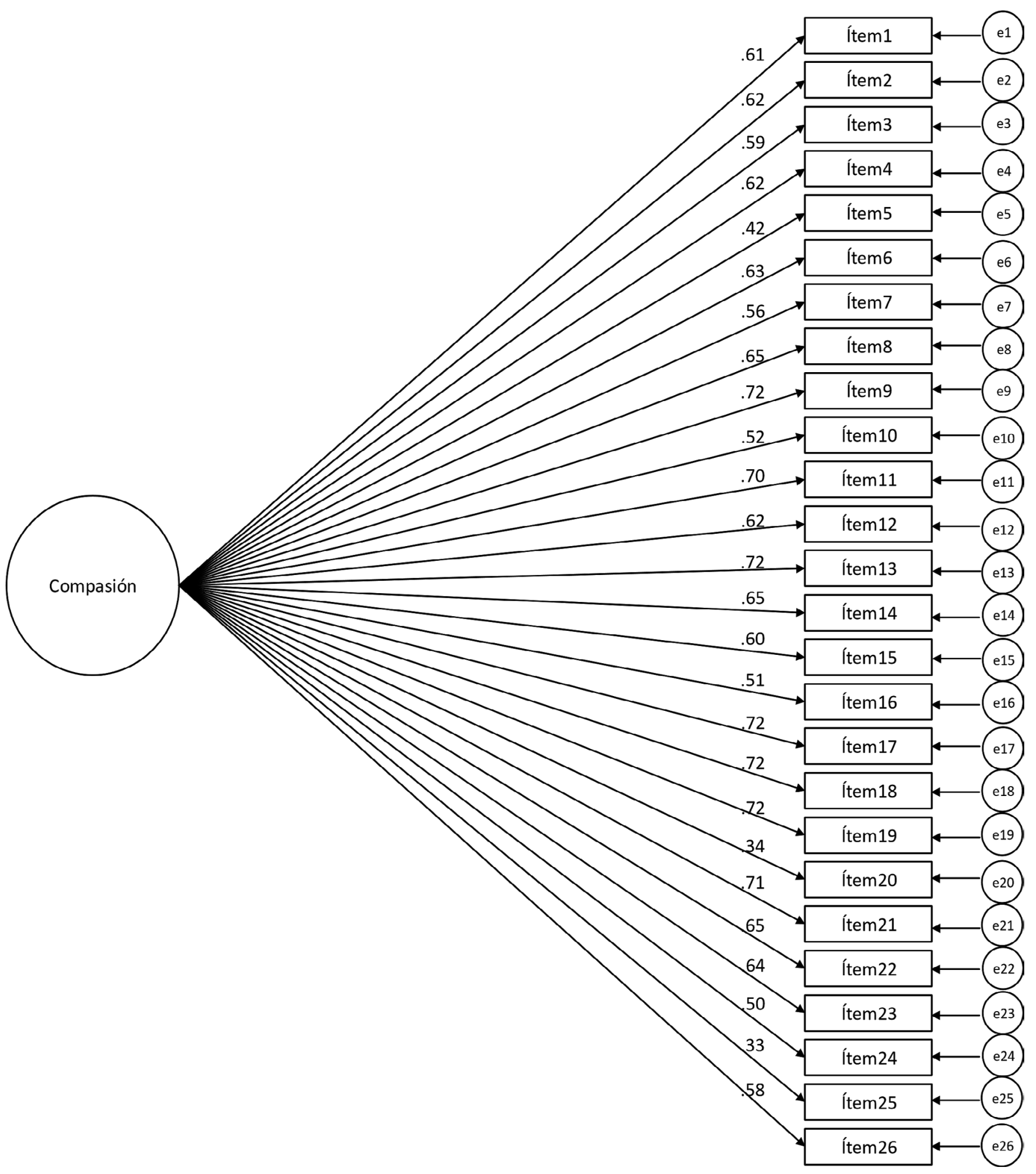

Figura 2

Modelo 2, versión unidimensional de la COOL de 26 ítems. 
Tabla 2

Ajuste de los modelos de medición de la COOL evaluados mediante AFC.

\begin{tabular}{lccccccccc}
\hline \multicolumn{1}{c}{ Modelo } & $\chi \mathbf{2}$ & $\boldsymbol{g} \boldsymbol{l}$ & $\boldsymbol{p}$ & $\chi \mathbf{2} / \boldsymbol{g} \boldsymbol{l}$ & GFI & AGFI & RMSEA & AIC & CI 90\% \\
\hline 1 (bifactorial) & 729.288 & 293 & $<.001$ & 2.489 & .868 & .842 & .062 & 845.288 & $.057-.068$ \\
2 (unidimensional) & 548.335 & 293 & $<.001$ & 1.87 & .890 & .868 & .048 & 664.335 & $.041-.054$ \\
\hline
\end{tabular}

Nota. $\chi^{2},=$ chi-cuadrada; $g l=$ grados de libertad, $p=$ significación; $\chi^{2} / g l=$ chi cuadrada sobre los grados de libertad; RMSEA = Error de aproximación de la media cuadrática; GFI = índice de bondad de ajuste; AGFI = índice de bondad de ajuste corregido; AIC = criterio de información de Akaike; IC 90\% = Intervalos de confianza al $90 \%$.

Se calcularon varios índices de ajuste, tal como recomienda la literatura (Hu \& Bentler, 1999; Tanaka, 1993) entre ellos el estadístico chi-cuadrado $\left(\chi^{2}\right)$ dividido por los grados de libertad, una de las más utilizadas, aunque es una medida muy afectada por el tamaño muestral (Bollen, 1989; Hayduk, 1987; Jöreskog \& Sörbom, 1982). En cuanto a su interpretación, se considera que un cociente de 4 es un ajuste razonable, (Wheaton, Muthén, Alwin, \& Summers, 1977), mientras que aquellos valores cercanos a 2 , son considerados como muy buenos (Tabachnick \& Fidell, 2007). Siguiendo las recomendaciones de otros autores (Browne \& Crudeck, 1993) se ha considerado también el promedio de los residuales estandarizados (RMSEA) debido a que proporciona un mejor índice de ajuste, y otros indicadores de ajuste relativo como el índice de bondad de ajuste (GFI) y el índice de bondad de ajuste corregido a los grados de libertad del modelo (AGFI). Estos índices se encuentran entre los más utilizados, ya que están menos afectados por el tamaño de la muestra (Bentler, 1990). Generalmente se considera que hay un buen ajuste cuando los índices de bondad de ajuste se encuentran en valores $>.90 \mathrm{y}$ RMSEA < .08 (Hu \& Bentler, 1999). También se ha calculado el criterio de información de Akaike (AIC) el cual indica una mayor parsimonia en el modelo que obtenga el menor valor; en este caso se observó un índice inferior en el modelo número 2 (unidimensional; Akaike, 1987).
Para evaluar la consistencia interna de la escala se calculó el coeficiente alfa de Cronbach, y se obtuvo un valor de $\alpha=.93$ (95\% CI .87-.99). La correlación entre ambas dimensiones fue de $r$ $=.77(95 \% \mathrm{CI}=.72-.80)$.

Para evaluar la validez convergente de la prueba, se realizó una correlación $r$ de Pearson entre las dimensiones del IRI y la COOL. Los resultados indicaron una correlación positiva y significativa entre ambas escalas $(r=.58 ; p=.000$; $[95 \% \mathrm{CI}=.45-.68])$.

\section{Discusión}

Considerando los beneficios y el impacto positivo que tiene la compasión en la vida de las personas, en los últimos años, en diferentes países se han estudiado y buscado métodos a fin de incrementarla (Gilbert \& Procter, 2006; Hurley, 2014; Jazaieri et al., 2012; Neff \& Germer, 2013; Reddy et al., 2012). En este sentido, sería de gran relevancia poder diseñar y aplicar un programa de intervención en nuestro contexto, destinado a la promoción de la compasión. Para evaluar la eficacia de un programa de intervención con este objetivo, es necesario contar con una medida válida y confiable del constructo. En este contexto, resulta necesario adaptar y validar un instrumento que lo mida de forma adecuada en nuestro medio, entendiendo que un constructo no necesariamente 
se expresa de igual forma en diferentes contextos y países (Fernández et al., 2011). De este modo el objetivo del presente estudio fue traducir, adaptar y validar la escala The Compassion of Others' Lives scale (COOL; Escala de Compasión hacia Otros) de Chang et al. (2014) en adolescentes y adultos jóvenes argentinos.

En cuanto a los resultados del funcionamiento de los ítems de la versión traducida y adaptada, se pudo observar que todos los ítems que la componen fueron discriminativos, lo que indica que permiten diferenciar entre quienes presentan mayores y menores niveles de compasión.

En cuanto a la validez de estructura interna factorial, a diferencia de la propuesta de los autores de la prueba, quienes teóricamente diferencian en la escala dos subdimensiones (empatía y alivio del sufrimiento), en este estudio se observó que si bien ninguno de los dos modelos presenta un ajuste óptimo a los datos, el modelo que presenta un mejor ajuste parcial a los datos observados fue el unidimensional. Los valores comparativos entre ambos modelos, considerando los valores de $\chi^{2} / g l$, GFI, AGFI y el criterio de Akaike (AIC), fueron mejores para el modelo unidimensional.

Una reciente versión turca (Coskun et al., 2017) validada en profesionales de la salud también encontró, a partir de un análisis factorial exploratorio, una estructura bidimensional, replicando los factores empatía y alivio del sufrimiento. Sin embargo, en nuestro contexto, como mencionamos anteriormente, ambas dimensiones se fusionaron en un único factor: compasión. Posiblemente esto se deba a la alta correlación que se presentó, como se mencionó anteriormente, entre las dos supuestas dimensiones de la escala, como así también a la alta correlación observada entre todos los ítems (ver coeficiente alfa de Cronbach). Es esperable que para que se produzca un acto de compasión sea necesario no solamente notar, o bien, ser sensible a la necesidad ajena, sino también ser movido a aliviar el sufrimiento de quien lo padece. Si bien es posible estudiar los componentes de la compasión como aspectos teóricamente diferenciables, ambos procesos estarían estrechamente unidos en la manifestación de dicho acto, pudiendo por esta razón unirse al ser estudiados empíricamente.

Respecto a la confiabilidad de la versión adaptada de la escala, tal como se indicó anteriormente, el coeficiente alfa de Cronbach indicó una muy alta consistencia interna (Campo-Arias \& Oviedo, 2008).

En cuanto a las evidencias de validez convergente del instrumento, se evaluaron junto a la COOL, dos dimensiones de la empatía, muy cercanas a la compasión: la preocupación empática y la toma de perspectiva. Los resultados hallados coinciden con otras investigaciones donde también se observó una correlación positiva entre la preocupación empática y la compasión (Hwang et al., 2008) como así también entre la empatía y la toma de perspectiva en relación a la compasión (Pommier \& Neff, 2010).

En síntesis, si bien la versión traducida y adaptada de la COOL presentó una satisfactoria consistencia interna y evidencias de validez convergente adecuadas, el AFC mostró un ajuste parcial a los datos. En la comparación de los modelos propuestos, el modelo unidimensional presentó índices relativamente más adecuados que el modelo bidimensional. En este sentido, este trabajo implica un avance vinculado a la medición de este constructo en adolescentes y adultos jóvenes de nuestro contexto, abriendo paso a nuevas investigaciones que permitan verificar los resultados encontrados hasta el momento. Avanzar en esta línea permitiría contar con un instrumento plausible de ser utilizado en el ámbito de la investigación, permitiendo evaluar la relación entre la compasión de los adolescentes y adultos jóvenes y diversas variables psicológicas (emocionales, sociales y 
cognitivas). Asimismo, permitiría evaluar la efectividad de programas destinados a promover y fortalecer este importante constructo psicológico en diversos ámbitos de la psicología aplicada ( $\mathrm{p}$. ej., educativa, comunitaria, entre otras).

\section{Referencias}

Akaike, H. (1987). Factor analysis and AIC. Psychometri$k a, 52(3), 317-332$. doi: 10.1007/bf02294359

Arbuckle, J. L. (2008). Amos 17.0. 2 (Version 17) [Software and Manual]. Crawfordville, FL: Amos Development Corporation.

Bentler, P. M. (1990). Comparative fit indexes in structural models. Psychological Bulletin, 107(2), 238-246. doi: 10.1037/0033-2909.107.2.238

Bollen, K. A. (1989). A new incremental fit index for general structural equation models. Sociological Methods \& Research, 17(3), 303-316. doi: 10.1177/0049124189017003004

Browne, M. W., \& Crudeck, R. (1993). Alternative ways of assessing model fit. En K. A. Bollen \& J. S. Long (Eds.), Testing Structural Equation Models, (pp. 136162). Newbury Park, CA: Sage.

Burnell, L., \& Agan, D. L. (2013). Compassionate care: Can it be defined and measured? The development of the Compassionate Care Assessment Tool. International Journal of Caring Sciences, 6(2), 180-187. Recuperado de http://www.internationaljournalofcaringsciences.org

Campo-Arias, A., \& Oviedo, H. C. (2008). Propiedades psicométricas de una escala: La consistencia interna. Revista de Salud Pública, 10(5), 831-839. doi: 10.1590/s0124-00642008000500015

Caprara, G. V., Steca, P., Zelli, A., \& Capanna, C. (2005). A new scale for measuring adults' prosocialness. $E$ uropean Journal of Psychological Assessment, 21(2), 77-89. doi: 10.1027/1015-5759.21.2.77

Chang, J.-H., Fresco, J., \& Green, B. (2014). The development and validation of the Compassion of Others'
Lives Scale (the COOL Scale). International Journal of Humanities and Social Science, 4(5), 33-42. Recuperado de http://www.ijhssnet.com

Coskun, H., Kavakli, O., Babayigit, M., \& Chang, J.-H. (2017). The psychometric evaluation of the Turkish version of the Compassion of Others' Lives Scale (the COOL). International Journal of Caring Sciences, 10(2), 637-646. Recuperado de http://internationaljournalofcaringsciences.org

Cuello, M. I., \& Oros, L. (Noviembre, 2014). Empatía y agresividad fisica y verbal en la infancia tardía. Trabajo presentado en VI Congreso Internacional de Investigación y Práctica Profesional en Psicología. XXI Jornadas de Investigación. Décimo Encuentro de Investigadores en Psicología del MERCOSUR. Facultad de Psicología. Universidad de Buenos Aires. Recuperado de http://jimemorias.psi.uba.ar

Davis, M. H. (1983). Measuring individual differences in empathy: Evidence for a multidimensional approach. Journal of Personality and Social Psychology, 44(1), 113-126. doi: 10.1037/0022-3514.44.1.113

Díaz-Alzate, M. V., Arbeláez-Gómez, L. M., \& David-Manco, O. T. (2015). La familia como espacio de socialización política: Prácticas de crianza que forman a niños y niñas en cuidado, reconocimiento y compasión (Tesis de maestría) . Facultad de Ciencias Sociales y Humanas, Universidad de Manizales, Colombia.

Fernández, A., Pérez, E., Alderete, A. M., Richaud, M. C., \& Fernández-Liporace, M. (2011). ¿Construir o adaptar tests psicológicos? Diferentes respuestas a una cuestión controvertida. Revista Evaluar, 10(1), 60-74. Recuperado de https://revistas.unc.edu.ar/index.php/revaluar

Gibbons, P., Flores de Arévalo, H., \& Mónico, M. (2004). Assessment of the factor structure reliability of the 28 item version of the General Health Questionnaire (GHQ-28) in El Salvador. International Journal of Clinical and Health Psychology, 4(2), 389-398. Recuperado de http://www.aepc.es 
Gilbert, P., \& Procter, S. (2006). Compassionate mind training for people with high shame and self-criticism: Overview and pilot study of a group therapy approach. Clinical Psychology \& Psychotherapy, 13(6), 353-379. doi: 10.1002/cpp.507

Goetz, J. L., Keltner, D., \& Simon-Thomas, E. (2010). Compassion: An evolutionary analysis and empirical review. Psychological Bulletin, 136(3), 351-374. doi: 10.1037/a0018807

Haidt, J. (2003). The moral emotions. En Davidson, R. J., Scherer, K. R., \& Goldsmith, H. H. (Eds.). Handbook of Affective Sciences, (pp. 852-870). Oxford: Oxford University.

Hayduk, L. A. (1987). Structural equation modeling with LISREL. Essentials and advances. Baltimore, MD: The Johns Hopkins University.

Hu, L.-T., \& Bentler, P. M. (1999). Cutoff criteria for fit indexes in covariance structure analysis: Conventional criteria versus new alternatives. Structural Equation Modeling: A Multidisciplinary Journal, 6(1), 1-55. doi: 10.1080/10705519909540118

Hurley, W. (2014). Enhancing a positive school climate with compassion and analytical selective-focus skills (COMPASS). Journal of Education and Practice, 5(7), 1-15. Recuperado de http://iiste.org

Hwang, J. Y., Plante, T., \& Lackey, K. (2008). The development of the Santa Clara Brief Compassion Scale: An abbreviation of Sprecher and Fehr's Compassionate Love Scale. Pastoral Psychology, 56(4), 421-428. doi: 10.1007/s11089-008-0117-2

IBM Corp. Released 2013. IBM SPSS Statistics for Windows, Version 22.0 [software de cómputo]. Armonk, NY: IBM Corp.

Jazaieri, H., Jinpa, T., McGonigal, K., Rosenberg, E. L., Finkelstein, J., Simon-Thomas, E., ... Goldin, P. R. (2012). Enhancing compassion: A randomized controlled trial of a compassion cultivation training program. Journal of Happiness Studies, 14(4), 11131126. doi: 10.1007/s10902-012-9373-z

Jöreskog, K. G., \& Sörbom, D. (1982). Recent developments in structural equation modeling. Journal of Marke- ting Research, 19(4), 404-416. doi: 10.2307/3151714 Klos, C., Lemos, V., \& Chang, J. H. (agosto, 2017). Psychometric performance of the "Compassion of Others Live's Scale" in Argentine adolescents. Comunicación interactiva presentada en el $125^{\circ}$ Congreso Anual de la American Psychological Association (APA). Washington DC, Estados Unidos.

Kroll, J., \& Egan, E. (2004). Psychiatry, moral worry, and the moral emotions. Journal of Psychiatric Practice, 10(6), 352-360. doi: 10.1097/00131746-200411000-00003

Lazarus, R. S. (1991). Emotion and adaptation. New York, NY: Oxford University.

Lazarus, R. S., \& Lazarus, B. N. (1996). Passion and reason: Making sense of our emotions. New York, NY: Oxford University.

Lilius, J. M., Worline, M. C., Dutton, J. E., Kanov, J. M., \& Maitlis, S. (2011). Understanding compassion capability. Human Relations, 64(7), 873-899. doi: 10.1177/0018726710396250

Martins, D., Nicholas, N. A., Shaheen, M., Jones, L., \& Norris, K. (2013). The development and evaluation of a compassion scale. Journal of Health Care for the Poor and Underserved, 24(3), 1235-1246. doi: 10.1353/hpu.2013.0148

Mercadillo, R. E., \& Díaz, J. L. (2013). Neuroscience and ethnography: An interdisciplinary revision and a cognitive proposal based on compassion research in Mexico. International Journal of Psychological Research, 6(Suplemento Especial), 94-108. doi: 10.21500/20112084.723

Mercadillo, R. E., Díaz, J. L., \& Barrios, F. A. (2007). Neurobiología de las emociones morales. Salud Mental, 30(3), 1-11. Recuperado de http://www.revistasaludmental.mx

Miller, K. I. (2007). Compassionate communication in the workplace: Exploring processes of noticing, connecting, and responding. Journal of Applied Communication Research, 35(3), 223-245. doi: 10.1080/00909880701434208 
Montero, I., \& León, O. G. (2007). A guide for naming research studies in psychology. International Journal of Clinical and Health Psychology, 7(3), 847-862. Recuperado de http://www.aepc.es

Muñiz, J., Elosua, P., \& Hambleton, R. K. (2013). Directrices para la traducción y adaptación de los tests: Segunda edición. Psicothema, 25(2), 151-157. Recuperado de http://www.psicothema.com

Muñiz-Fernández, J. (2000). Adaptación de los test de unas culturas a otras. Metodología de las Ciencias del Comportamiento, 2(2), 129-149.

Neff, K. D., \& Germer, C. K. (2013). A pilot study and randomized controlled trial of the Mindful Self-Compassion program. Journal of Clinical Psychology, 69(1), 28-44. doi: 10.1002/jclp.21923

Ostrosky-Solís, F., \& Vélez-García, A. E. (2008). Neurobiología de la sensibilidad moral. Revista Neuropsicología, Neuropsiquiatría y Neurociencias, 8(1), 115-126. Recuperado de https://dialnet.unirioja.es

Poch, C., \& Vicente, A. (2010). La acogida y la compasión: Acompañar al otro. En J. C. Mèlich \& A. Boixader (coords.), Los márgenes de la moral: Una mirada ética a la educación (pp. 86-100). Barcelona: GRAÓ.

Pommier, E. A., \& Neff, K. D. (2010). Compassion Scale. PsycTESTS Dataset. doi: 10.1037/t10177-000

Reddy, S. D., Negi, L. T., Dodson-Lavelle, B., Ozawa de Silva, B., Pace, T. W. W., Cole, S. P., Craighead, L. W. (2012). Cognitive-based compassion training: A promising prevention strategy for at-risk adolescents. Journal of Child and Family Studies, 22(2), 219-230. doi: 10.1007/s10826-012-9571-7

Regner, E. (2009). Compasión y gratitud, emociones empáticas que elicitan las conductas prosociales. En M. C. Richaud \& J. E. Moreno (Comp.), Investigación en Ciencias del Comportamiento. Avances Iberoamericanos (pp. 281-298). Buenos Aires: CIIPME - CONICET.

Richaud de Minzi, M. C. (2008). Evaluación de la empatía en población infantil argentina. Revista de Investigación en Psicología, 11(1), 101-115. doi: 10.15381/ rinvp.v11i1.3880
Richaud de Minzi, M. C., Lemos, V., \& Oros, L. (2003). Adaptación del NEO-PI-R a la Argentina. Primera parte: Diferencias entre el "español" y el "argentino" en el NEO-PI-R: Su influencia sobre la validez constructiva. Psicodiagnosticar, 13, 27-45.

Saavedra, B. A., Mola, D. J., Gancedo, K. M., Belaus, A., \& Reyna, C. (2013). Una revisión sistemática sobre emociones morales, dilemas sociales y cooperación. Trabajo presentado en XIV Reunión Nacional y III Encuentro Internacional de la Asociación Argentina de Ciencias del Comportamiento. Facultad de Psicología de la Universidad Nacional de Córdoba, Córdoba, Argentina.

Servera, M., \& Cardo, E. (2006). Children Sustained Attention Task (CSAT): Normative, reliability, and validity data. International Journal of Clinical and Health Psychology, 6(3), 697-707. Recuperado de http:// www.aepc.es

Sprecher, S., \& Fehr, B. (2005). Compassionate love for close others and humanity. Journal of Social and Personal Relationships, 22(5), 629-651. doi: 10.1177/0265407505056439

Tabachnick, B. G., \& Fidell, L. S. (2007). Usando estadisticas multivariadas ( $5^{\text {a }}$ ed.). Nueva York, NY: Allyn \& Bacon.

Tanaka, J. S. (1993). Multifaceted conceptions of fit in structural equation models. Sage Focus Editions, 154, 10-10.

Wheaton, B., Muthén, B., Alwin, D. F., \& Summers, G. F. (1977). Assessing reliability and stability in panel models. Sociological Methodology, 8, 84-136. doi: $10.2307 / 270754$

Yubero, S., \& Larrañaga-Rubio, M. E. (2002). Concepción del voluntariado desde la perspectiva motivacional: Conducta de ayuda vs. altruismo. Pedagogía Social. Revista Interuniversitaria, 9, 27-39. Recuperado de http://www.redalyc.org/articulo. oa? id=135018332002 